\title{
Risk Measurement of the American Basket Options Based on the Least Square Monte Carlo Simulation Approach
}

\author{
Qiang Li, Xueling Zhu
}

School of Finance, Guizhou University of Finance and Economics, Guiyang 550025, China

\section{基于最小二乘蒙特卡洛模拟的美式篮子期权的风险度量 \\ 李 强, 朱雪玲 \\ 贵州财经大学金融学院 (贵州省金融研究院), 贵阳 550025, 中国}

\begin{abstract}
This article applies a simple yet powerful LSM approach for approximating the value of American basket options by simulation. The primary objective is to compare basket Index prices derived from different noise processes. As alternatives, the Brownian motion benchmark is compared to noise processes driven by Gaussian and Student's t copulas, and then three distinct regions of the piecewise distribution is estimated with a semi-parametric probability distribution with generalized Pareto tails. Once we have simulated sample paths, options are priced by the least squares regression method of Longstaff \& Schwartz. With the result of American basket put options on stock indices, VaR can be measured by the Latin Hypercubs sampling techniques approximation. The result shows that Latin Hypercubs sampling techniques can be applied to China's stock index option risk measurement.
\end{abstract}

Keywords: American basket options, Least Square Mento Carlo simulation, Value at Risk, Latin Hypercubs samping techniques

\section{摘要}

本文采用一种简约而有效的 LSM 方法对美式篮子期 权进行模拟定价。首先通过布朗模型、多元高斯和 $\mathrm{t}$ Copula 模型对世界主要股指组合的价格噪声过程进 行比较, 然后采用半参数方法对股指组合的累积分布
函数划分为三个区域进行估计, 再运用帕累托分布拟 合尾部极值, 确立了模拟的样本路径, 美式篮子期权 的价格可由 Longstaff \& Schwartz. 的 LSM 方法获得, 最后将得到的美式篮子看跌期权作为输入变量, 采用 拉丁方抽样技术对中国上证综指期权的市场风险进 行了度量, 得到了一些有意义的结论。

关键词: 美式篮子期权; 最小二乘蒙特卡洛模拟; 在 险价值; 拉丁方抽样技术

\section{1. 引言}

期权 (Option) 指在未来某一特定的时间, 以某一约定 的价格买卖另一种金融证券的证券。期权实际上赋予 期权持有者一种选择权利, 如美式期权就给予持有者 在有效期内可任意执行的权利。由于美式期权具有可 提前执行, 在金融领域把美式期权的定价问题归结为 最佳执行边界问题。期权定价理论最重要的问题之一 是评估美式期权的最佳执行问题, 特别是当对于期权 的价格的影响多于一个因子时, 美式期权的最佳执行 是最具挑战的难题之一。在高维期权定价方面, 蒙特 卡洛由于其计算工作量与问题的维数呈线性增长而 不像其它定价方法那样呈指数增长, 因而更具优势。 Boyle $\mathrm{e}^{[1]}$ 最先把蒙特卡洛方法引入到金融分析领域并 应用于期权定价研究以来, 蒙特卡洛作为有效的数值 分析方法广泛应用于金融衍生工具的定价方面。

近些年来, 随着金融创新不断地深入, 美式衍生 工具和具有非标准复杂特性的新型金融衍生证券大 量出现, 蒙特卡洛模拟由于自身的特点在实际运用过 程中受到了一定程度的限制, 如蒙特卡洛模拟的前向 模拟特性, 在对具有后向迭代搜索特征的美式期权的 
Risk Analysis and Crisis Response in Big Data Era (RAC-16)

应用过程中受到了一定的限制; 在对依赖路径期权的 应用上, 也产生了较大的方差。国内外学者们针对上 述局限性, 探索出了一些实用的解决办法。Till $\mathrm{y}^{[2]}$ 在Bossaerts ${ }^{[3]}$ 尝试运用模拟法对美式期权定价的基 础上, 采用蒙特卡洛模拟对支付红利的美式期权进行 定价, 提出束划分方法。Broadle和Glasserman ${ }^{[4]}$ 针 对Tilly方法存储和运算工作量大的不足, 提出对一 维期权的盈利收益空间进行划分, 使得划分处理独立 于标的变量数的束方法。Grant ${ }^{[5]}$ 等将具有后向搜索 特征的动态规划思想融入蒙特卡罗方法框架中, 有效 地解决了美式期权的估价问题, 在计算和数据存储量 及精度上都较前述的方法有一定的改进。特别要提及 的是Longstaff Schwartz ${ }^{[6]}$ 提出的最小二乘蒙特卡洛 模拟方法 ( the Least Square Monte Carlo Approach, LSM), 它完善了Carrier ${ }^{[7]}$ 和Tsitsiklis s ${ }^{[8]}$ 基于线性回归所有样本路径所有时点的不足, 同时也 弥补了传统差分技术不能应用于路径依赖和多因子 情形的缺陷。另外, 该方法在计算和数据存储量方面 均有较大突破。国内学者马俊海 ${ }^{[9-10]}$ 较好综述了蒙特 卡洛模拟对美式期权的定价计算方法。基于数值改进 方法对美式期权研究的有: 张德飞等 ${ }^{[11]}$ 建立具有连续 分红和交易成本的美式看跌期权的数值定价模型, 姜 礼尚 ${ }^{[12]}$ 等深入研究了美式期权定价二叉树方法的收 玫性问题以及多资产的美式期权定价问题。唐明琴 ${ }^{[13]}$ 介绍了美式期权的蒙特卡洛模拟法定价理论, 并在此 基础上推导出的线性回归蒙特卡洛模拟法定价公式 及在实际中的应用。杨海军 ${ }^{[14]}$ 采用加权最小二乘法进 行回归, 通过数值试验得出WLSQM方法比LSM方法估计 效果较优。从上述文献中可以看到国内的研究主要集 中在数值方法和国外相关方法的介绍及应用上, 对金 融衍生工具市场风险的度量, 尤其是美式篮子股指期 权风险的度量研究还很少见。特别是采用LSM方法得 到美式篮子看跌期权的价格, 再运用拉丁方抽样技术 对中国股指期权进行风险度量, 就笔者掌握的文献而 言, 本文研究尚属首次。

本文在前面学者研究的基础上, 首先通过布朗模 型、多元高斯和 t Copula 模型对世界主要股指篮子 组合的价格噪声过程进行比较, 然后采用半参数方法 对股指的累积分布函数划分为三个区域进行估计, 再 运用帕累托分布拟合尾部极值, 确立了模拟的样本路 径, 美式篮子期权的价格可由 LSM 方法进行定价得 到, 进而将得到的美式篮子看跌期权作为输入变量, 采用拉丁方抽样技术对中国指数期权的市场风险进 行了度量。

\section{2. 检验美式篮子期权定价理论基础}

\section{1. 基于多标的资产的伊藤引理和 Black-scholes} 方程

篮子期权的定价需要把多标的资产期权定价问题转 化为一维问题来处理, 并假定每个标的资产价格情况 都服从几何布朗运动 (Geometric Brownian Motion, GBM):

$$
d S_{i}=\mu_{i} S_{i} d t+v_{i} S_{i} d W_{i}
$$

其中, $d W_{i}$ 是一个维纳过程, $\mu_{i}$ 和 $v_{i}$ 分别是标的 资产价格 $S_{i}$ 的期望收益和波动率。假定 $S_{1}, \ldots, S_{n}$ 为 $N$ 个标的资产过程相关, $K=K\left(S_{1}, \ldots, S_{n}, t\right)$ 是 $N+1$ 个 $S_{1}, \ldots, S_{n}$ 和 $t$ 的函数, 标的资产 $S_{i}, S_{j}$ 的相关系数为 $\rho_{i j}$ 。令 $f\left(S_{1}, \ldots, S_{i}, \ldots, S_{n} t\right)$ 为标的资产与时间的函数, 通 过Itô 引理, $f(\bullet)$ 可表示为:

$$
d f=\left[\frac{\partial f}{\partial t}+\frac{1}{2} \sum_{i, j=1}^{n} \rho_{i} v_{i} V_{j} S_{i} S_{j} \frac{\partial^{2} f}{\partial S_{i} \partial S_{j}}\right] d t+\sum_{i=1}^{n} \frac{\partial f}{\partial S_{i}} d S_{i}
$$

根据 $\Delta$ 对冲原理，选取 $\Delta_{i}$ 份股价指数 $S_{i}(i=1, \ldots, n)$, 使之与期权 $K$ 进行对冲, 形式为:

$$
\Pi=K\left(S_{1}, \ldots, S_{n}, t\right)-\sum_{i=1}^{n} \Delta_{i} S_{i}
$$

通过建立套期保值组合，使得其在时间间隔 $(t, t+d t)$ 上是无风险的。由多维随机过程的 Itô 引理, 则有 (4):

$$
\begin{aligned}
& d \Pi=d K-\sum_{i=1}^{n} \Delta d S_{i}-\sum_{i=1}^{n} \Delta, S q, d t
\end{aligned}
$$

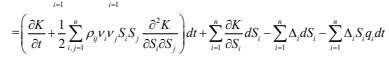

其中, $q_{i}$ 为股价指数 $S_{i}$ 的红利率, 若时间间隔区 无分红, 则 $q_{i}$ 为零.

$$
\begin{aligned}
& d \Pi=r \Pi d t=r\left(K-\sum_{i=1}^{n} \Delta_{i} S_{i}\right) d t \quad \Delta_{i}=\frac{\partial K}{\partial S_{i}} \\
& \text { 代入上式 (4), 并消去 } d t, \text { 得 (5): } \\
& \quad \frac{\partial K}{\partial t}+\frac{1}{2} \sum_{i, j=1}^{n} \rho_{i j} v_{i} v_{j} S_{i} S_{j} \frac{\partial^{2} K}{\partial S_{i} \partial S_{j}}+\sum_{i=1}^{n}\left(r-q_{i}\right) S_{i} \frac{\partial K}{\partial S_{i}}=r K
\end{aligned}
$$

此方程即为多标的资产的Black-scholes方程。

\section{2 美式篮子期权定价模型和线性互补模型}

基于 B-S 期权定价模型主要应用于欧式期权的 定价, 但大部分上市期权均为复杂的可提前执行的美 式期权, 在金融领域把美式期权的定价问题归结为最 佳执行边界或转化为依赖于时间的线性互补问题。下 面重点探讨美式看跌期权最佳执行边界的确定问题。

由于美式篮子期权具有提前执行的特性, 故无套 利原则将保证从投资组合所获取的收益应小于无风 
险收益, 即 $d \Pi<r \Pi d t$ 。对于美式篮子看跌期权, 相应有 (6):

$$
\frac{\partial K}{\partial t}+\frac{1}{2} \sum_{i, j=1}^{n} \rho_{i j} V_{i} V_{j} S_{i} S_{j} \frac{\partial^{2} K}{\partial S_{i} \partial S_{j}}+\sum_{i=1}^{n}\left(r-q_{i}\right) S_{i} \frac{\partial K}{\partial S_{i}} \leq 0, S_{i} \geq 0
$$

在任何时间 $t$, 都存在一个股价指数的价格 $\tilde{S}(t)$, 当即期市场价格 $S \leq \tilde{S}(t)$ 时, 存在 $K(S, t)=\max (X-S, 0)$, 而当 $S>\tilde{S}(t)$ 时, $K(S, t) \geq \max (X-S, 0)$ 。最佳执行边界 $\tilde{S}(t)$ 与损益函数 $\max (X-S, 0)$ 连接点的斜率为 -1 。美式篮子 看跌期权价格 $K(S, t)$ 是式 (7) 最佳执行边界的解:

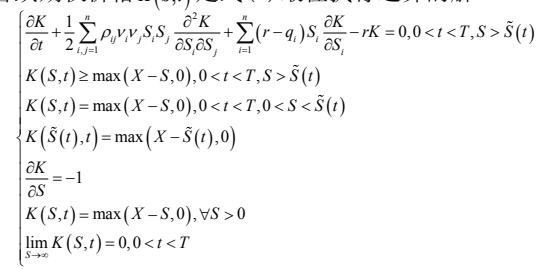

为了求出美式篮子期权的价格, 还必须解决 $n$ 维 线性互补问题。

假设在有效期内任一时刻 $t$ 执行期权的收益为

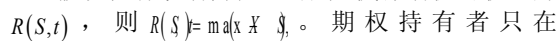
$K(S, t) \leq R(S, t)$ 时, 才会考虑提前执行, 最终导致该 时刻的期权价值 $K(S, t)=R(S, t)$ 。当期权价值 $K(S, t)>R(S, t)$, 至有效期期满执行则相当于一个欧 式期权, 可通过 $B-S$ 方程进行求解。因此美式篮子期 权在有效期 $0<t<T$ 内可归结为:

$\int \frac{\partial K}{\partial t}+\frac{1}{2} \sum_{i, j=1}^{n} \rho_{i j} v_{i} v_{j} S_{i} S_{j} \frac{\partial^{2} K}{\partial S_{i} \partial S_{j}}+\sum_{i=1}^{n}\left(r-q_{i}\right) S_{i} \frac{\partial K}{\partial S_{i}}-r K \leq 0, K(S, t)=R(S, t), 0<S<\tilde{S}(t)$ $\left\{\frac{\partial K}{\partial t}+\frac{1}{2} \sum_{i, j=1}^{n} \rho_{i j} V_{i} V_{j} S_{i} S_{j} \frac{\partial^{2} K}{\partial S_{i} \partial S_{j}}+\sum_{i=1}^{n}\left(r-q_{i}\right) S_{i} \frac{\partial K}{\partial S_{i}}-r K=0, K(S, t) \geq R(S, t), S>\tilde{S}(t)\right.$ 由上, 若 $K, \partial K / \partial t$ 连续, 美式篮子期权的线性互补问题 用下列式 (8):

$\left\{\begin{array}{l}\left(\frac{\partial K}{\partial t}+\frac{1}{2} \sum_{i, j=1}^{n} \rho_{i j} v_{i} v_{j} S_{i} S_{j} \frac{\partial^{2} K}{\partial S_{j} \partial S_{j}}+\sum_{i=1}^{n}\left(r-q_{i}\right) S_{i} \frac{\partial K}{\partial S_{i}}-r K\right)(K(S, t)-R(S, t))=0 \\ \text { 在 } K(S, t)-R(S, t) \geq 0 \text { 下, 上式成立 } \\ K(S, t)=\max (X-S, 0) \\ \lim _{S \rightarrow \infty} K(S, t)=0\end{array}\right.$

\section{3. 美式篮子期权定价的最小二乘蒙特卡洛方法}

蒙特卡洛方法是 Boyle $\mathrm{e}^{[1]}$ 最先引入到金融分析领域并 应用于期权定价研究。蒙特卡洛模拟在对美式篮子期 权进行定价时, 需要用有限个离散时间步骤数的提前 执行概率来表示连续时间内的可能提前执行决策。但 是蒙特卡洛模拟的前向模拟特性, 在对具有后向迭代 搜索特征的美式篮子期权的应用过程中受到了一定 的限制, 由 Longstaff 和 Schwartz ${ }^{[6]}$ 提出的 LSM 效
果较好, 它通过后向迭代搜索方式得到美式篮子期权 的价格。该方法关键在于使用最小二乘法来估计连续 支付给期权持有者的条件期望回报率。

LSM 的计算原理: 它通过量化美式期权的最优执 行策略, 以此来确定美式期权在各个时点上的价值。 假设美式期权在 $K$ 个离散交易时点 $0<t_{1} \leq t_{2} \leq t_{3} \leq \cdots \leq t_{k}=T$ 被执行, 当 $K$ 足够大时, 可以 有效地渐进期权的精度。现在考虑在任一执行时点的 最优停时, 令 $C\left(\omega, t_{j} ; t_{k}, T\right)$ 表示 $t_{j}$ 时点期权收益现金 流, $\omega$ 表示第 $\tau$ 条模拟样本路径; $r(\omega, t)$ 表示无风险 贴现利率, 是时间的函数; $F\left(\omega ; t_{k}\right)$ 表示 $t_{k}$ 时点期权 的持有期望收益; $\Psi_{t_{k}}$ 表示 $t_{k}$ 时点所有相应标的资产 价格信息。期权持有人在 $t_{k}$ 时点, 即刻执行收益已知, 继续持有收益未知。由无套利定价原理可知, 持有收 益应为下一交易时点 $t_{j}$ 期权收益以无风险利率 $r(\omega, t)$ 贴现到 $t_{k}$ 时的现金流。因此, $t_{k}$ 时点的期权 持有收益的条件期望函数, 式 (9):

$$
F\left(\omega ; t_{k}\right)=E\left[\sum_{j=k+1}^{k} \exp \left(-\int_{t_{k}}^{t_{j}} r(\omega, t) d t\right) C\left(\omega, t_{k} ; t_{j}, T\right) \mid \Psi_{t_{k}}\right]
$$

实证表明当标资产的隐含期权价格符合马尔科夫 过程时, 拟合的条件期望函数就可由多个不同阶的 Laguerre多项式线性组合而成, 其式 (10) 如下:

$$
\left\{\begin{array}{l}
L_{0}(X)=\exp (-X / 2) \\
L_{1}(X)=\exp (-X / 2)(1-X) \\
L_{2}(X)=\exp (-X / 2)\left(1-2 X+X^{2} / 2\right) \\
L_{n}(X)=\exp (-X / 2) \frac{e^{X}}{n !} \frac{d^{n}}{d X^{n}}\left(X^{n} e^{-X}\right)
\end{array}\right.
$$

由此定义可知, $F\left(\omega ; t_{k-1}\right)$ 可表示为:

$$
F\left(\omega, t_{k-1}\right)=\sum_{j=0}^{\infty} a_{j} L_{j}(X)
$$

其中 $a_{j}$ 为常数。

\section{4. 实证分析}

\section{1 样本的选取}

本文样本选取从 2005 年 8 月 8 日至 2011 年 3 月 15 日的美国 S\&P500 指数、德国 DAX 指数、法国 CAC40 指数、中国香港 HSI 指数 (中国上证 SSCI 指 数)、英国 FTSE100 指数和日经 Nikkei225 指数的日 收盘指标作为期权的标的物, 样本容量为 1359 组数 据, 采用 3 个月期限的 Euribor 利率, 假设篮子组合 持有每一指数的一个单位, 且在任何时刻组合的值等 于篮子里每一指数值的和。样本数据来源于雅虎财经 
Risk Analysis and Crisis Response in Big Data Era (RAC-16)

网。所有指数均采取 对 数收益率即 $R_{t}=\ln \left(p_{t} / p_{t-1}\right)$ 。由于美式篮子期权的最终收益依 赖于到期日标的指数的当日收盘价, 故上证综指指数 的现值取 2011 年 3 月 15 日上证综指日收盘价, 即 $S=2896.26$ 。无风险利率取 2010 年 1 月 1 日的一年 期存款利率 $r=2.25 \%$, 不考虑上证综指数的红利增长 率。股指期权有效期设定为三个月，通过 LSM 方法拟 合上证综指历史数据得到其波动率为 0.2748 。同时 分别设定上证综指一点为 100 元和协定价格为 2700 。

图 1 可知, 左图为世界主要指数的日历史数据进 行统一规范化处理。右图为上证综指 (绿色曲线) 整 体上显示与世界主要股指运行并不一致。然后根据世 界主要指数样本收益序列的边缘分布, 通过两阶段的

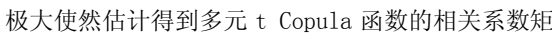

阵。表 1 略, 考虑了上证综指的一篮子指数中, 其他 指数间的相关性比不考虑上证综指的相关性得到了 增强。t Copula 的自由度参数也从 3.7 提高到了 5.8 。

图 2 可知, 左图显示对上证综指的累计分布函数 中间部分采用高斯核平滑密度估计上证综指的经验 或非参数分布, 然而其在拟合上下尾部时表现较差。 考虑采用 GPD 通过极大使然估计对上下尾部超出帕 累托阈值的极值进行拟合, 显然给定模型较好拟合了 上证综指的边缘分布。右图显示为在得到 $\mathrm{t} \mathrm{Copula}$ 参数的估计值后, 可以对联合均匀分布进行模拟。

由表 2 (略), 包含上证综指的美式篮子指数期 权的价格低于不包含上证综指对应的期权价格。在 LSM 方法下, 随着模拟次数的增加, 美式篮子期权价 格的精度在增加, 从 Copula 模型得到的美式篮子看 跌期权价格与从布朗模型得到的误差值不超过 $1 \%$ 。
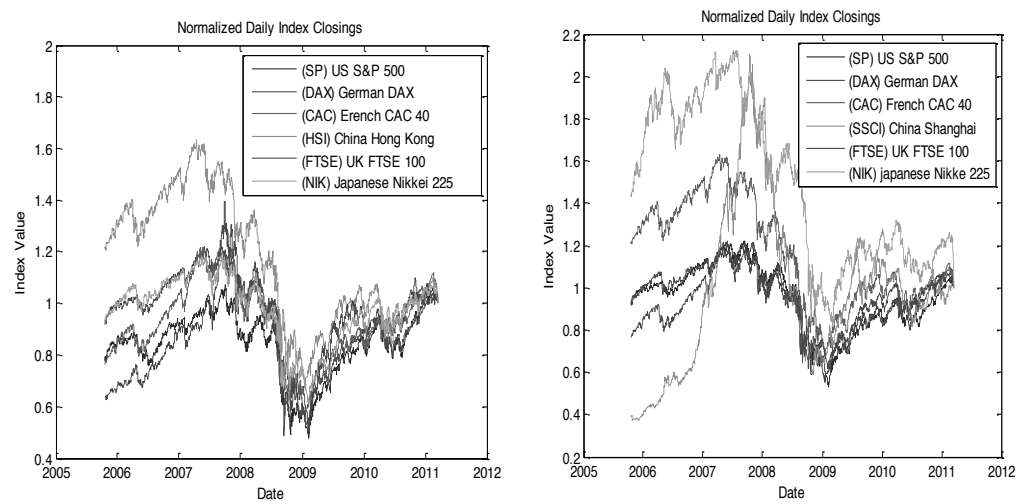

图 1. 世界主要指数日收盘价格运行趋势
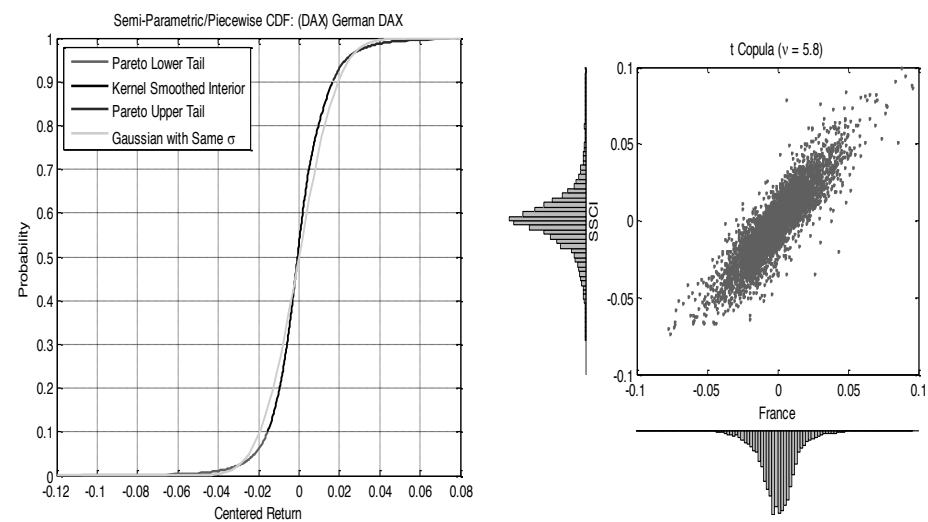

图 2. 上证综指的分段函数和上证与 CAC 指数基于 $\mathrm{t} \mathrm{Copula}$ 的散点图 
Risk Analysis and Crisis Response in Big Data Era (RAC-16)

最后, 基于上证综指的 $\mathrm{t}$ Copula 美式看跌指数 期权价格 12.0150 , 拟构建两个组合: 即持有上证综 指的多头资产和购买美式看跌期权的组合 $\mathrm{A}$ 和仅持 有上证综指的多头资产组合 $B$ 。然后对组合 $A$ 和 $B$ 的 收益进行对比, 同时采用@RISK 软件的拉丁方抽样技 术抽样 1000 次得到相应的分位数, 以此为基础对上 证综指美式期权的市场风险进行度量。

基于拉丁方抽样技术 1000 次抽样中，在有无购 买美式看跌期权的组合 $\mathrm{A}$ 和 $\mathrm{B}$ 的收益对比中, 若无购 买置信度 $95 \%$ 的 $\mathrm{VaR}$ 则为上证综指-的 $15.5 \%$, 即 $\mathrm{VaR}$ 为 -448.92 点, 按设定每点 100 元计 VaR 为 -44892 元; 若购买, 则 VaR 则相应下降为 $-7.2 \%$, 即 VaR 为 -20853 元。同时还注意到, 若没有购买美式看跌期 权, 则上证综指还存在最大损失为 $-32.7 \%$ 的可能, 即 相应 VaR 为 -94708 元。同时在可能获取的最大收益 对比上, 组合 A 仅比组合 B 存在减少 $0.7 \%$, 即相应 值为 2027 元。

从图 3 可知, 图示为上证综指以拉丁方抽样 1000
区域进行估计, 再运用帕累托分布拟合尾部极值, 确 立了模拟的样本路径, 美式篮子期权的价格可由LSM 方法进行定价得到, 最后将得到的美式篮子看跌期权 作为输入变量, 采用拉丁方抽样技术对中国指数期权 的市场风险进行了度量。

通过计算和分析, 我们可以得到如下结论: (1)在 中国指数融入世界主要股指的篮子组合中, 样本期间 其他指数间的相关性得到了增强, 美式篮子指数期权 的价格相应地降低。(2)在基于美式篮子看跌期权的风 险度量中, 以中国指数为例, 保护性看跌股指期权的 组合的 $\mathrm{VaR}$ 仅为 $-7.2 \%$, 而无购买欧式看跌期权的资 产组合则存在损失其价值为 $-32.7 \%$ 的可能性, 同时前 者还大幅地降低了VaR, 提高了投资现金流的比例, 尽管购买美式篮子看跌期权付出了相应成本, 但是进 行了有保护性的组合在规避和控制风险方面显然更 具优势。随着中国金融市场创新型产品的不断推出以 及定价难度的提升, 蒙特卡洛模拟和拉丁方抽样技术 作为优越的数值分析方法, 在金融衍生工具定价和风

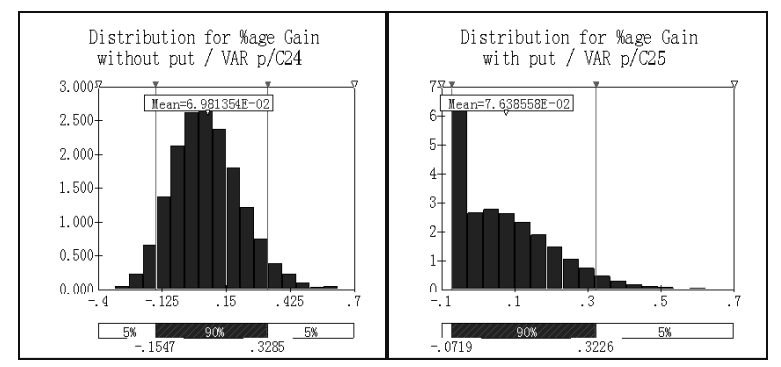

图 3 有无购买美式看跌期权的上证综指组合收益分布对比

次为例, 有无购买美式看跌期权的组合 $A$ 和 $B$ 的收益分 布, 若没有购买美式看跌期权, 组合 $\mathrm{B}$ 存在损失其价 值为 $-32.7 \%$ 的可能。反之若购买美式看跌期权, 则组 合 $\mathrm{A}$ 的平均收益为 $0.0763 \%$, 高于无购买欧式看跌期权 的组合B的 $0.006575 \%$ 。原因在于上证综指在选取的样 本区间呈现巨幅震荡, 尽管购买美式看跌期权付出了 一定成本, 显然有保护性的组合 $\mathrm{A}$ 在规避和控制风险 方面更具优势。

\section{5 结束语}

目前, 国内基于 $V a R$ 方法对美式篮子期权进行风 险度量的文献胗胗无几，本文基于VaR方法和期权的 相关理论, 选取世界主要股指组合作为分析对象, 首 先通过布朗模型和多元高斯和 t Copula模型对世界 主要股指的篮子组合的价格噪声过程进行比较, 然后 采用半参数方法对股指的累积分布函数划分为三个
险管理方面将有更广泛的应用。

\section{Acknowledgements}

This study was supported by Guizhou Province of Philosophy and Social Fund Project (No.15GZYB32).

\section{致谢}

本研究得到了贵州省哲社基金项目（15GZYB32） 的资助。

\section{参考文献}

[1] Boyle P. Options: a Monte Carlo method. Journal of Financial Economics, 1977, (4) : 323- 33.

[2] Tilly J. Valuing American opt ions in a path simulation model. Transactions of the Society of 
Risk Analysis and Crisis Response in Big Data Era (RAC-16)

Actuaries, 1993, 45: 83- 104.

[3] Bossaerts P. Simulation estimators of option early exercise. Working paper, Carnegie Mellon Universty. 1989

[4] Broadie M. and Glasserman P. Pricing Americanstyle securitiesusing simulation. Journal of Economics Dynamics and Control, 1997, 21:13231352

[5]Longstaff F A. , and Schwartz E S. Valuing American opt ions by simulation: a simple least - squares approach. The Review of Financial Studies, 2001, $14(1): 113-147$

[6]Ma Jun-hai and Zhang Wei. Analysis on recent applications of Mont e Carlo simulation method for pricing financial derivatives. Journal of Engineering Management, 2000, 14(2) : 47- 50

[7]张德飞,崔向照和赵金娥. 美式看跌期权的数值解 法.兰州大学报.2009,6(45): 104-106 转 115 .

[8]Jiang.L.S..Analysis of pricing American options on the maximum (minimum) of two Risk assets. Interface \& Free Boundaries.2002,4:27-46

[9]唐明琴.美式期权的Mento Carlo模拟法定价理论应 用.广东金融学院学报.2007,(5):36-40.

[10]杨海军,雷杨.基于加权最小二乘拟蒙特卡罗的美 式期权定价[J].系统工程学报.2008,(5):532-538.

[11]Abdullah Alam. Crisis Transmission: Global Financial Crisis. Journal of Risk Analysis and Crisis Response. 2012,2(11): 157-165. 